\title{
Impaired exercise parameters in pediatric heart transplant recipients: Comparison of biatrial and bicaval techniques
}

\begin{abstract}
Pahl E, Sundararaghavan S, Strasburger JF, Mitchell BM, Rodgers S, Crowley D, Gidding SS. Impaired exercise parameters in pediatric heart transplant recipients: Comparison of biatrial and bicaval techniques. Pediatr Transplantation 2000: 4: 268-272. (C) Munksgaard, 2000

Abstract: The exercise performance of pediatric heart transplant recipients and the effects of bicaval anastomosis were studied in 19 children using a Bruce protocol. Although all children had decreased exercise capacity and heart rates when compared with normals, the bicaval anastomosis patients had similar endurance and peak heart rates as the standard biatrial group.
\end{abstract}

\section{Elfriede Pahl' ${ }^{1}$, Sreekanthan \\ Sundararaghavan ${ }^{1}$, Janette $F$. Strasburger ${ }^{1}$, Brett M. Mitchell', Sherrie Rodgers', Dennis Crowley ${ }^{2}$ and Samuel S. Gidding ${ }^{1}$}

${ }^{1}$ Departments of Pediatrics and Medicine,

Northwestern University Medical School, Chicago, Illinois, USA, ²Department of Pediatrics, University of Michigan, Ann Arbor, Michigan, USA

Key words: pediatric heart transplantation treadmill exercise testing - biatrial anastomosis - bicaval anastomosis.

Elfriede Pahl MD, 2300 Children's Plaza, Box \#21, Chicago, IL 60614, USA

Tel.:+1 773-880-4553

Fax:+1 773-880-8111

E-mail: epahl@nwu.edu

Accepted for publication 5 January 2000
Although there have been many studies of adult transplant recipients assessing exercise capacity (1-4), there are only a few studies in pediatric heart transplant recipients $(5,6)$. Hsu et al. have shown that transplant recipients have decreased exercise capacity on a bicycle ergometer protocol (5). However, there are no published studies using an exercise treadmill protocol in pediatric patients. This study characterized the exercise response of children who have undergone cardiac transplantation, using a treadmill protocol. In addition, the impact of the type of surgical anastomosis on exercise performance was analyzed.

\footnotetext{
Abbreviations: b.p.m., beats per minute; DBP, diastolic blood pressure; ECG, electrocardiogram; LVEDP, left ventricular enddiastolic pressure; RA, right atrium; RQ, respiratory quotient; RV, right ventricle; SBP, systolic blood pressure; VO2, oxygen consumption.
}

Presented in part at The American Heart Association 71st Scientific Sessions, Dallas, Texas, November 1998.

\section{Patients and methods}

Study group

Exercise tests carried out on pediatric heart transplant recipients between 1988 and 1996 at two centers were included in this study, provided that patients met the following criteria: (i) $\geq 6 \mathrm{yrs}$ of age; (ii) $\geq 1$ month post rejection episode; (iii) normal left ventricular function on a recent echocardiogram; and (iv) no evidence of transplant coronary artery disease by angiography. The first exercise test following transplantation was used for analysis. There were 19 patients from two centers (16 males and 3 females), as described in Table 1. Two cohorts were identified based on surgical technique. Bicaval anastomosis was performed in five recipients using a previously reported technique (7) and the remainder had standard biatrial technique. Medical records were reviewed to obtain demographics and medications, to exclude recent rejection, and to determine hemodynamics, resting cardiac index 
and ejection fraction at cardiac catheterization performed closest in time to the exercise stress test.

\section{Exercise protocol}

Cardiac transplant recipients underwent a symptom-limited exercise test on a treadmill (Quinton, Bothell, WA, USA) using the Bruce protocol modified by a standing recovery phase (8). Standing resting heart rate, baseline 12-lead ECG, and pulmonary measurements were recorded prior to starting exercise. Heart rate, blood pressure, breath-by-breath metabolic measurements (Medgraphics, St. Paul, MN, USA), and continuous 12-lead ECG were monitored throughout the test. Studies were analyzed for endurance time, resting and peak heart rate, resting and peak blood pressure, metabolic variables (peak oxygen consumption, RQ etc.), and occurrence of arrhythmias or ischemic changes.

\section{Statistics}

Descriptive analyses were performed from variables of interest. Individual performance was compared with established normative data (9). Patients were subgrouped by type of surgery (biatrial vs. bicaval anastomosis) and the Wilcoxon rank sum test was used for group comparison. A p-value of $<0.05$ was considered significant.

\section{Results}

Patient demographics

As a whole, patient age ranged from 7.8 to $21 \mathrm{yr}$ (mean 15.6 $\pm 3.5 \mathrm{yr}$ ). Time from transplantation was $0.04-7.6 \mathrm{yr}$ (mean $3.0 \pm 2.4 \mathrm{yr}$ ). Immunosuppression was cyclosporin A-based in all. Oral prednisone was used in 18 of the 19 patients (mean dose $0.19 \pm 0.07 \mathrm{mg} / \mathrm{kg} /$ day). Antihypertensive therapy included angiotensin inhibitors

Table 1. Patient demographics

\begin{tabular}{lrrr}
\hline & \multicolumn{1}{c}{ Biatrial } & \multicolumn{1}{c}{ Bicaval } & p-value \\
\hline Number of patients & 14 & 5 & \\
Age $(\mathrm{yr})$ & $14.8 \pm 3.4$ & $17.7 \pm 3.2$ & $\mathrm{NS}$ \\
Height $(\mathrm{cm})$ & $157 \pm 18$ & $160 \pm 15$ & $\mathrm{NS}$ \\
Weight $(\mathrm{kg})$ & $53.9 \pm 21$ & $54.5 \pm 12$ & $\mathrm{NS}$ \\
Body mass index $\left(\mathrm{kg} / \mathrm{m}^{2}\right)$ & $21.3 \pm 5.8$ & $21.3 \pm 3.4$ & $\mathrm{NS}$ \\
Years since transplant & $3.4 \pm 2.7$ & $2.0 \pm 0.5$ & $\mathrm{NS}$ \\
LVEDP (mmHg) & $7.86 \pm 1.9$ & $8.00 \pm 4.1$ & $\mathrm{NS}$ \\
Cardiac index (rest; L/kg/min) & $4.3 \pm 1.9$ & $3.9 \pm 1.0$ & $\mathrm{NS}$ \\
Ejection fraction (\%) & $80.1 \pm 5.5$ & $68 \pm 2.0$ & $<0.05$ \\
\hline
\end{tabular}

LVEDP, left ventricular end-diastolic pressure. and/or calcium channel blockers in 16 patients; none were taking beta-blockers at the time of stress testing.

The bicaval group tended to have undergone transplantation more recently than the biatrial group; however, there were no significant differences in age, height, weight, hemodynamics, or resting cardiac index at catheterization (see Table 1). Prednisone dose expressed in $\mathrm{mg} / \mathrm{kg}$ body weight did not differ between the two surgical groups.

\section{Exercise parameters}

Metabolic and pulmonary data were analyzed in eight patients to determine if maximal exertion was given, and all eight achieved maximum effort, as determined by $\mathrm{RQ}>1.1$, ventilatory exchange of $>50 \mathrm{~L} / \mathrm{min}$, and a respiratory rate of $>40$ breaths/min. Reasons for termination of the test included fatigue, leg fatigue, and dyspnea. Total endurance time for the cohort was 6.0-12.6 min (mean 8.6 $\pm 1.9 \mathrm{~min}$ ), which was less than the 10th percentile in 15/19 patients $(79 \%)$ (see Table 2). Two patients achieved endurance levels in the 10-25th percentile.

Heart rate data are shown in Table 2. The resting heart rates of the post-transplant patients (mean 100 16 beats/min, range 62-131) were significantly higher than the age- and sexpredicted mean in the normal population (mean $80 \pm 7$ beats $/ \mathrm{min}$, range $72-102, \mathrm{p}<0.001$ ). With exercise, the mean peak heart rate in transplant patients was $153 \pm 21$ beats/min (range 113-202), which was lower than the age- and sex-predicted mean in $18 / 19$ patients (mean $200 \pm 3$ beats/min, p $<0.001)$. The heart rate response by exercise stage is shown in Fig. 1. The heart rates were higher than normal in stage 1 , commensurate with normals in stage 2 and lower than normal by stage 3 . The increase in mean heart rates from one stage to another was significantly lower in transplant recipients than normals.

Resting and peak blood pressure values are shown in Table 2. In 10/19 patients, the peak systolic blood pressure was attained in recovery. Peak oxygen consumption was measured in 16 patients and ranged from 17.3 to $44.7 \mathrm{~mL} / \mathrm{kg} / \mathrm{min}$ (mean 28.0 \pm 8.5 ), which represents a decrease compared with average $\mathrm{VO} 2$ in normal children (range $45-60 \mathrm{~mL} / \mathrm{kg} / \mathrm{min}$ ) (10). Peak oxygen pulse for the post-transplant group was $11.0 \pm 3.6 \mathrm{~mL} /$ beat.

Arrhythmias occurred in three patients: two had isolated premature ventricular contractions and one developed a junctional rhythm. Both patients with premature beats had a biatrial 
anastomosis and the patient with junctional rhythm had a bicaval anastomosis.

\section{Biatrial and bicaval comparison}

Exercise parameters were compared between the two surgical groups and are presented in Table 2. There were no significant differences in exercise duration or peak heart rate between the two groups (see Fig. 1). Moreover, none of the exercise parameters was significantly different between the two groups. The biatrial group had a greater exercise duration, however it did not reach the significance level (biatrial $9.0 \pm 1.9 \mathrm{~min}$ vs. bicaval $7.4 \pm 1.1 \mathrm{~min}, \mathrm{p}=0.086$ ). In addition, peak oxygen consumption was similar among patients who had biatrial or bicaval anastomosis. Oxygen pulse measurements were analyzed at peak exercise and were not different between the groups (biatrial $11.2 \pm 3.9 \mathrm{~mL} /$ beat vs. bicaval $10.5 \pm 3.7 \mathrm{~mL} /$ beat, $\mathrm{p}=0.268$ ).

\section{Discussion}

In this study, we examined the cardiac work capacity of pediatric heart transplant recipients. We demonstrated that their response to exercise includes a diminished chronotropic response, diminished endurance time and diminished peak VO2. There were no significant arrhythmias or other adverse responses.

Oxygen consumption measurements and heart rates have been shown to be higher in children when treadmill protocols are utilized compared with bicycle protocols (11). This probably results from the child's ability to reach aerobic exhaustion prior to onset of local muscle fatigue. This is corroborated when our findings are compared with the peak heart rates achieved in the bicycle ergometer study of pediatric heart recipients by

Table 2. Comparison of exercise parameters

\begin{tabular}{lrrr}
\hline & Biatrial $(\mathrm{n}=14)$ & Bicaval $(\mathrm{n}=5)$ & p-value \\
\hline Treadmill time (min) & $9.0 \pm 1.9$ & $7.4 \pm 1.1$ & $\mathrm{NS}$ \\
Resting heart rate (b.p.m.) & $101 \pm 16$ & $99 \pm 10$ & $\mathrm{NS}$ \\
Peak heart rate (b.p.m.) & $155 \pm 23$ & $150 \pm 16$ & $\mathrm{NS}$ \\
Resting SBP (mmHg) & $124 \pm 15$ & $128 \pm 7$ & $\mathrm{NS}$ \\
Resting DBP (mmHg) & $82 \pm 11$ & $73 \pm 19$ & $\mathrm{NS}$ \\
Peak SBP (mmHg) & $142 \pm 28$ & $160 \pm 13$ & $\mathrm{NS}$ \\
Peak DBP (mmHg) & $74 \pm 18$ & $69 \pm 12$ & $\mathrm{NS}$ \\
Peak V02 (mL/kg/min) & $24 \pm 9.6$ & $30 \pm 7.6$ & $\mathrm{NS}$ \\
Peak oxygen pulse (mL/beat) & $11.2 \pm 3.9$ & $10.5 \pm 3.7$ & $\mathrm{NS}$ \\
Peak respiratory quotient & $1.22 \pm 0.1$ & $1.18 \pm 0.1$ & $\mathrm{NS}$ \\
Peak respiratory rate (breaths/min) & $48 \pm 10$ & $48 \pm 5$ & $\mathrm{NS}$ \\
Ventilatory exchange (L/min) & $80 \pm 15$ & $65 \pm 18$ & $\mathrm{NS}$ \\
\hline Presented & & & \\
\hline
\end{tabular}

$\overline{\text { Presented as mean } \pm \text { SD. b.p.m., beats per minute; DBP, diastolic blood pressure; }}$ SBP, systolic blood pressure; VO2, oxygen consumption.
Hsu et al. Despite higher heart rates elicited from treadmill testing, poor chronotropic response was still evident at stage 3 of the protocol in transplant recipients.

Cardiac denervation and deconditioning posttransplant have been identified as factors responsible for submaximal exercise performance in adults (4). Animal studies have shown that, despite a delayed heart rate response and decreased peak heart rate, maximal cardiac output and VO2 were unaffected (12). Differences between animal studies and adult studies of exercise performance include (i) hemodynamic effects of immunosuppressive agents, particularly cyclosporin A $(13,14)$; (ii) steroid-induced myopathy; and (iii) decreased skeletal muscle mass (15). All of these may contribute to poor performance in pediatric heart transplant recipients, in addition to low levels of conditioning. Despite these limitations, a small percentage of patients achieved normal exercise performance post-transplant. Thus, we speculate that performance could be improved with training, as was demonstrated in adult studies that reported a significant improvement in exercise capacity with short-term endurance training (16, 17).

In many centers, the biatrial anastomosis has been replaced by the bicaval anastomosis for heart transplantation. Animal studies reported in 1998 confirmed preserved sinus rhythm in $10 / 10$ dogs who had bicaval vs. only $1 / 10$ animals transplanted by biatrial technique; furthermore, the standard group had distorted RV geometry with RV dilatation, and abnormal septal motion, whereas the bicaval group had normal RV size and shape (18). Studies done in adults have demonstrated the following hemodynamic benefits: decreased tricuspid regurgitation, improved right-atrial dimensions, and decreased use of pacemakers (19-23). Furthermore, Freimark and colleagues found that right- and left-atrial function were significantly depressed with the biatrial technique and markedly improved with the bicaval; they speculated that these beneficial effects could improve allograft exercise performance (19). Our findings did not support an adult study that demonstrated that recipients with bicaval anastomosis had greater exercise endurance times (22), as we showed that those with biatrial anastomosis actually had slightly higher exercise duration although peak VO2 was not significantly different. We recognize that the small number of patients in the bicaval group may have failed to show a significant difference. In an adult study, despite unfavorable factors in the biatrial group such as higher RA 
Fig. 1. Mean heart rates at different stages of Bruce protocol: comparison between transplant recipients $(\boldsymbol{\nabla})$ and normals $(\square)$.

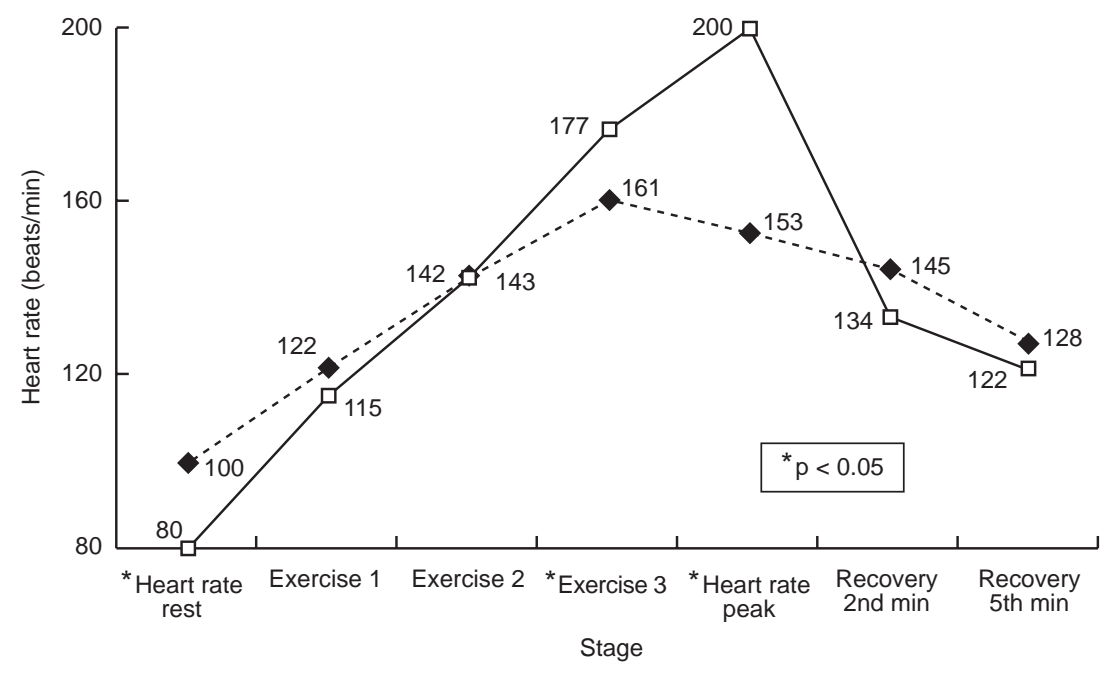

pressure, a higher incidence of tricuspid insufficiency, and asynchronous RA contraction on echocardiography, exercise capacity was comparable to the bicaval group (22). Bainbridge and colleagues recently reported a randomized study of 58 adult transplant recipients, 29 had biatrial and 29 had bicaval, and confirmed our findings of similar peak oxygen consumption and peak heart rates (23).

In summary, pediatric heart transplant patients have lower exercise capacities and peak heart rates than normal children despite favorable resting hemodynamics and left ventricular ejection fractions. Further investigation should address whether endurance training with a cardiac rehabilitation program would be beneficial to improve exercise capacity in this cohort, and whether any improvement in exercise parameters can be identified in bicaval transplant recipients.

\section{Acknowledgments}

This study was supported in part by a grant from the Dr Scholl Foundation (grant number 953020) and the Stone Heart Fund. We are grateful to Dr Chen, Director of Biostatistics at University of Illinois at Chicago and Ms. Tammy Tarsa for secretarial assistance.

\section{References}

1. Kavanaugh T, Yacoub MH, Mertens DJ, Kennedy J, Campbell RB, Sawyer P. Cardiorespiratory responses to exercise training after orthotopic cardiac transplantation. Circulation 1988: 1: 162-171.

2. Michael M, Givertz L, Hartley H, Collucci WS. Longterm sequential changes in exercise capacity and chronotropic responsiveness after cardiac transplantation. Circulation 1997: 96: 232-237.

3. Ehrman JK, Keteyian SJ, Levine AB, et al. Exercise stress tests after cardiac transplantation. Am J Cardiol 1993: 71: 1372-1373.
4. Renlund DG, Taylor DO, Ensley RD, et al. Exercise capacity after heart transplantation: influence of donor and recipient characteristics. J Heart Lung Transplant 1996: 15: 16-24.

5. Hsu DT, Garofono RP, Douglas JM, et al. Exercise performance after pediatric heart transplantation. Circulation 1993: 88: 238-242.

6. Nixon PA, Fricker FJ, Noyes BE, Webber SA, Orenstein DM, Armitage JM. Exercise testing in pediatric heart, heart-lung and lung transplant recipients. Chest 1995: 107: 1328-1335.

7. Sievers HH, Weyand M, Kraatz EG, Bernhard A. An alternative technique for orthotopic cardiac transplantation, with preservation of the normal anatomy of the right atrium. Thorac Cardiovasc Surg 1991: 39: 70-72.

8. BRUCE RA. Exercise testing of patients with coronary artery disease. Ann Clin Res 1971: 3: 323-330.

9. Cumming GR, Everatt D, Hastman L. Bruce treadmill test in children: normal value in a clinic population. Am J Cardiol 1978: 41: 69-75.

10. Freedson PS, Goodman TL. Measurement of oxygen consumption. In: Rowland TW, ed. Pediatric Laboratory Testing. Clinical Guidelines. Illinois: Human Kinetic Publishers, 1993: 91-113.

11. Rowland TW. Aerobic exercise testing protocols. In: Rowland TW, ed. Pediatric Laboratory Testing. Clinical Guidelines. Illinois: Human Kinetic Publishers, 1993: $19-41$.

12. Pope S, Stinson E, Daughters G, Schroeder J. Exercise responses of the denervated heart in long term cardiac transplant recipients. Am J Cardiol 1980: 46: 213-218.

13. Nixon JV, Rosenburg H, Romhilt D, Thompson JA, Hastillo A, Hess ML. Response to dynamic exercise of orthotopically transplanted human heart in men immunosuppressed with cyclosporine. Am J Cardiol 1989: 64: 401-403.

14. Plugfelder PW, Purves PD, Mckenzie FN, Kostuk WJ. Cardiac hemodynamics during supine exercise in cyclosporine treated orthotopic heart transplant recipients: assessment by radionuclide angiography. J Am Coll Cardiol 1987: 10: 336-341.

15. Lampert E, Mettauer B, Hoppeler H, Charloux A, Charpentier A, Lonsdorfer J. Structure of skeletal muscle in heart transplant recipients. J Am Coll Cardiol 1996: 28: 980-984.

16. Geny B, Saini J, Mettauer B. Effect of short term 


\section{Pahl et al.}

endurance training on exercise capacity, hemodynamics and atrial natriuretic peptide secretion in heart transplant recipients. Eur J Appl Physiol 1996: 73: 259-266.

17. Kavanaugh T. Physical training in heart transplant recipients. J Cardiovasc Risk 1996: 3: 154-159.

18. Bittner HB, Chen EP, Kendall SW, Biswas SS, Davis D, TRIGT PV. Right ventricular function in orthotopic total atrioventricular heart transplantation. J Heart Lung Transplant 1998: 17: 826-834.

19. Freimark D, Silverman JM, Aleksic I, et al. Atrial emptying with orthotopic heart transplantation using bicaval and pulmonary venous anastomoses: a magnetic resonance imaging study. J Am Coll Cardiol 1995: 25: 932-936.

20. Trento A, Taknenberg JM, Czer LSC, et al. Clinical experience with one hundred consecutive patients under- going orthotopic heart transplantation with bicaval and pulmonary venous anastomosis. J Thorac Cardiovasc Surg 1996: 112: 1496-1503.

21. Sievers HH, Leyh R, JahnKe A, et al. Bicaval versus atrial anastomosis in cardiac transplantation. J Thorac Cardiovasc Surg 1994: 108: 780-784.

22. Leyh RG, Jahnke A, Kraatz EG, Sievers HH. Cardiovascular dynamics and dimensions after bicaval and standard cardiac transplantation. Ann Thorac Surg 1995: 59: 1495-1500.

23. Bainbridge AD, Cave M, Roberts M, Casula R, Mist BA, Parameshwar J, Wallwork J, Large SR. A prospective randomized trial of complete atrioventricular transplantation versus ventricular transplantation with atrioplasty. J Heart Lung Transplant 1999: 18: 407-413. 